\title{
The digital patient
}

\author{
Timothy Bonnici, Lionel Tarassenko, David A Clifton and Peter Watkinson
}

\begin{abstract}
Despite efforts, the detection of patients who are deteriorating in hospital is often later than it should be. Several technologies could provide the basis of a solution. Recording of vital signs could be improved by both automated transmission of the measured parameters to an electronic patient record and the use of unobtrusive wearable monitors that track the patient's physiology continuously. Electronic charting systems could make the recorded vital signs readily available for further processing. Software algorithms could identify such patients with greater sensitivity and specificity than the existing, paperbased track-and-trigger systems. Electronic storage of vital signs also makes intelligent alerting and remote patient surveillance possible. However, the potential of these technologies depends strongly on implementation, with poor-quality deployment likely to worsen patient care.
\end{abstract}

KEYWORDS: Early warning score (EWS), emergency treatment, monitoring, patient safety, vital signs

\section{Introduction}

Over the past decade, there has been a considerable focus on the identification and response to unexpected clinical deterioration occurring in hospitalised patients. Much of this work has been prompted by studies demonstrating that many deteriorations are not detected in a timely fashion, leading to unplanned admission to the intensive care unit or to cardiac arrest, which would have been avoidable had appropriate care been instituted at an earlier stage. ${ }^{1}$ Factors contributing to avoidable cardiac arrests have been examined in several studies, ${ }^{2,3}$ most notably the National Confidential Enquiry into Patient Outcome and Death (NCEPOD) report in $2005 .{ }^{4}$ These studies identified a wide variety of systemic problems, ranging from failure to record patient vital signs with sufficient frequency to failure to respond appropriately to a patient identified as having deteriorated. Following National Institute for Health and Clinical Excellence (NICE) guidance, ${ }^{5}$ hospitals in the UK have implemented early warning scores (also called 'track-and-trigger' scores) to aid detection of the deteriorating patient on the general ward. Yet, despite these scores, late detection of such patients remains a problem. ${ }^{6}$

As part of its ongoing work to address this issue, the Royal College of Physicians recently published proposals for a National

Timothy Bonnici, clinical research fellow; Lionel Tarassenko, professor of electrical engineering; David A Clifton, junior research fellow; Peter Watkinson, honorary clinical lecturer

University of Oxford, UK
Early Warning Score (NEWS), ${ }^{7}$ advocating that it should be widely adopted across National Health Service (NHS) hospitals with the aspiration that improvements in patient care will occur through standardisation of alerting thresholds and escalation procedures. Implementation of NEWS will require hospitals to change observations charts, adjust escalation pathways and retrain staff. Therefore, this represents an opportunity to consider how recent technological developments could improve the identification of patients who are deteriorating.

As shown in Fig 1, systems for identification and treatment of patients who are deteriorating may be divided into the following components: devices to measure markers of abnormality (patient monitors recording vital signs); systems to record the collected data (often a paper chart); systems to analyse the recorded data (such as an Early Warning Scoring System); and systems to respond to the results of the analysis (the alerting and escalation pathways of a hospital). In this article, we provide an overview of the technological options for each component and how they might develop in the future. Although there is overlap with technologies used in intensive care environments, we focus primarily on systems applicable to Level 1 wards.

\section{Measurement of vital signs}

The existing standard of care in British hospitals is continuous monitoring in high dependency and intensive care areas and intermittent monitoring using 'spot-check' monitors on level 1 wards. In exceptional cases, the latter can be augmented by continuous monitoring using either portable bedside monitors or wireless telemetry devices on wards where wireless access points are installed.

The typical hardware used for recording vital signs on the level 1 ward can be improved in several ways. Introduction of spot-check monitors capable of wirelessly transmitting the recorded observations to an electronic charting system should lead to process improvements by eliminating transcription errors and speeding the process of recording observations. Wireless spot-check monitors can either be purchased as systems with integrated wireless networking cards or created by retrofitting existing monitors with an additional device to enable wireless transmission. ${ }^{8}$

An alternative strategy for improving the observation recording process is more widespread use of continuous (or near-continuous) recording of vital signs using multiparameter monitors, with any intermittent measurements, such as measurement of blood pressure, being triggered by the monitor. Theoretically, this should lead to detection of abnormal physiology at the earliest possible moment and reduce the incidence of observations being missed or being taken too infrequently. However, when this approach was trialled using existing bedside monitors in both medical and 


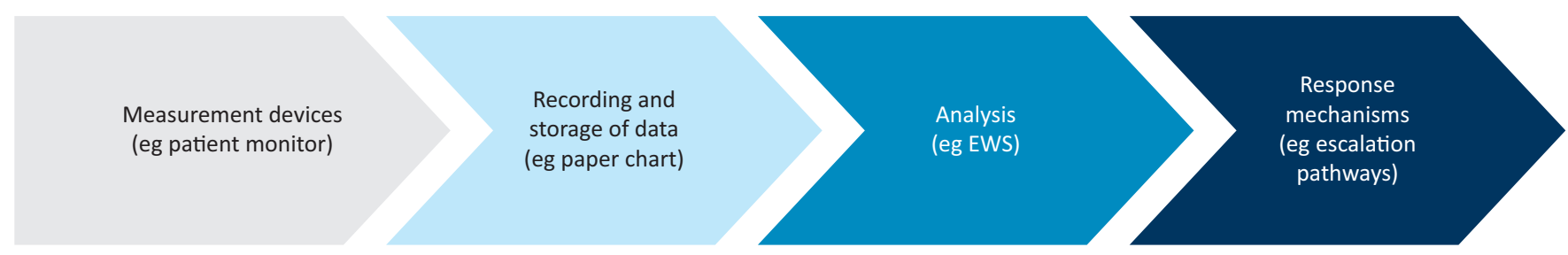

Fig 1. Generic components of a system to identify and respond to patients who are deteriorating in hospital. EWS = early warning score.

surgical populations, no benefit was found on intention-to-treat analysis. ${ }^{9}$ Strikingly, only $16 \%$ of patients assigned to automatic monitoring remained connected to their monitors for the entire 72 hour period mandated by the study protocol. The most common reasons cited for removal of monitoring were patient request and to enable patients to mobilise, clearly demonstrating the ergonomic inadequacy of conventional bedside monitors for long-term recordings in ambulatory patients. For continuous monitoring to provide benefit in a general ward population, equipment needs to be more comfortable and less restrictive.

In response to this challenge, several manufacturers have developed novel monitors. Wearable cardiac telemetry devices are already established in the hospital environment. However, most devices designed for in-hospital use rely on a radiofrequency network specific to the device to transmit the acquired data. Installing the requisite network access points is costly and ties the hospital to a single provider of telemetry. The devices themselves are bulky compared with some of the newer monitors under development (see below) and most are restricted to monitoring echocardiography (ECG), although a few can also record oxygen saturation. Such solutions do not remove the need for intermittent monitoring, because the other vital signs also need to be recorded.

The newer monitors on the market (examples of which are listed in Table 1) are generally closer to being wearable for prolonged periods and many record a more diverse set of physiological parameters, including motion (which can be used both to detect patient falls and to measure patient activity levels). Only one device available to date, the Sotera Wireless VisiMobile, is explicitly targeted at the hospital environment and claims to measure all five commonly recorded vital signs. The remainder are either designed to cater for outpatient monitoring or for short-term use in high-risk environments.

Unfortunately, these newer, wearable monitors are not yet suitable for widespread deployment as clinical monitoring devices in a hospital environment, partly because of lack of suitable supporting software optimised for use by clinical staff in a hospital environment and partly because of the design choices made when targeting them for out-of-hospital use. In a recent assessment of three novel monitors, none managed to record data consistently for $24 \mathrm{~h} .{ }^{10}$ However, given the considerable amount of interest in home monitoring using wearable monitors (the Department of Health spent over $\mathfrak{£} 30$ million funding the recent Whole System Demonstrator trial ${ }^{11}$ to assess the benefits of telehealth), it is likely that these systems will mature rapidly.
Wearable sensors are not the only monitoring technologies being explored. Sensors woven into clothing, ${ }^{12}$ printed on transfer tattoos ${ }^{13}$ and embedded into furniture ${ }^{14}$ are also being developed. Monitors need not be physically attached to patients in any way: non-contact sensing technologies include video cameras, ${ }^{15,16}$ Doppler radar $^{17}$ and ultra-wideband radar. ${ }^{18}$ Although such sensors are not currently sufficiently developed to enable mainstream use in a hospital environment, they do offer the promise of ubiquitous monitoring without impairment of patient mobility.

However, an ergonomic monitor that simply transmits data to a remote screen or stores the information locally is less useful than a wearable monitor connected to a system that stores the recorded data and makes it available for further analysis. The nature and potential benefits of analysis are discussed below. Such processing is greatly facilitated by the adoption of open standards for the storage and transmission of data. This has been widely recognised and consortia, such as the Continua Healthcare Alliance and Integrating the Healthcare Enterprise, have been formed by key industry players to promote the use of open standards. Their efforts are complemented by work done in the open-source community, a notable example being the OpenEHR project, whose standards for the storage, retrieval and exchange of patient data have been adopted by the NHS through the Connecting for Health program.

\section{Charting of vital signs}

Existing practice in most UK hospitals is to record the vital signs on a paper chart, with the nurse recording the observations, adding the scores for each vital sign parameter and then entering the overall early warning score on the chart. This approach has a risk of error in both assigning the correct score to each vital sign parameter and calculating the total score. ${ }^{19-21}$ Other well-described problems with the paper chart are poor legibility, inaccurate plotting and the possibility of misplacing or losing the chart. These flaws are frequently cited as reasons for the introduction of electronic charting systems. ${ }^{22}$

Such systems can be broadly divided into three categories: charting modules, which are part of an electronic patient record application; stand-alone charting systems; and charting systems that are designed to work solely with the wireless spot-check monitors described above.

Studies investigating the benefits of electronic systems report highly variable results, in part because their success or failure is 
defined in narrow terms, such as the speed of data entry or user acceptance, ${ }^{23}$ rather than from a broader clinical perspective (which in itself is only one of many pertinent perspectives ${ }^{24}$ ) and also because benefits measured in a non-clinical setting ${ }^{19}$ might not correspond to benefits on the ward. Furthermore, the introduction of electronic charting systems might have unintended consequences by paradoxically making data less easily accessible to nursing staff. ${ }^{25}$ Despite over two decades of development in this field, there are still many lessons to be learnt regarding the optimal design and functionality of such systems. Nevertheless, such systems do enable a transformative change in the way that vital sign data are used: storing the data electronically makes them available for more complex and varied processing than is afforded by calculation of an integer early warning score.

\section{Processing of vital signs}

Existing early warning scores are designed to be simplistic so as to facilitate calculation using pen and paper. This limits the sensitivity and specificity that they can hope to achieve. A completely electronic system enables integration of non-vital sign parameters (such as laboratory results) and patient-related factors (such as age and comorbidities) into a scoring system, ${ }^{26}$ as well as facilitating complex analysis of vital signs. Rather than assuming

Table 1. Examples of commercially available novel wearable sensors.

\begin{tabular}{|c|c|c|}
\hline Device & Form factor & Parameters recorded \\
\hline Intelens Aingeal ${ }^{54}$ & $\begin{array}{l}\text { Attaches via magnets to an } \\
\text { adhesive pad placed on the } \\
\text { chest wall }\end{array}$ & $\begin{array}{l}\text { ECG } \\
\text { Respiration via impedance pneumography } \\
\text { Skin surface temperature } \\
\text { Motion and activity via tri-axial accelerometer }\end{array}$ \\
\hline Equivital EQ02 LifeMonitor ${ }^{55}$ & $\begin{array}{l}\text { Worn in a small pocket in a } \\
\text { thoracic belt }\end{array}$ & $\begin{array}{l}\text { ECG } \\
\text { Respiration via resistive strain gauge } \\
\text { Skin surface temperature } \\
\text { Motion and activity via tri-axial accelerometer } \\
\text { Core temperature can be measured if patients have swallowed a core temperature pill } \\
\text { Optionally: } \\
\text { - pulse oximetry } \\
\text { - galvanic skin resistance (sweating) }\end{array}$ \\
\hline Sotera Wireless VisiMobile ${ }^{56}$ & $\begin{array}{l}\text { Wrist-worn module with ECG } \\
\text { leads connected to standard } \\
\text { chest electrodes }\end{array}$ & $\begin{array}{l}\text { ECG } \\
\text { Respiration rate via impedance pneumography and accelerometry } \\
\text { Skin surface temperature } \\
\text { Blood pressure } \\
\text { Pulse oximetry } \\
\text { Motion and activity via tri-axial accelerometer }\end{array}$ \\
\hline Zephyr Bio-Harness ${ }^{57}$ & Belt worn around the chest & $\begin{array}{l}\text { ECG } \\
\text { Respiration rate } \\
\text { Skin surface temperature } \\
\text { Motion and activity via tri-axial accelerometer }\end{array}$ \\
\hline Nonin WristOx ${ }_{2} 3150^{58}$ & $\begin{array}{l}\text { Wrist-worn module with a } \\
\text { conventional pulse oximetry } \\
\text { probe }\end{array}$ & Pulse oximetry (giving both oxygen saturation and heart rate) \\
\hline Isanys LifeTouch ${ }^{59}$ & $\begin{array}{l}\text { A small adhesive patch worn } \\
\text { on the chest }\end{array}$ & $\begin{array}{l}\text { ECG } \\
\text { Respiration rate derived from the ECG signal }\end{array}$ \\
\hline Proteus Raisin Patch ${ }^{60}$ & $\begin{array}{l}\text { A small adhesive patch worn } \\
\text { on the torso }\end{array}$ & $\begin{array}{l}\text { Heart rate (via ECG) } \\
\text { Motion and activity via tri-axial accelerometer } \\
\text { Skin surface temperature } \\
\text { Medication ingestion (requires patients to take an additional identifier pill, or for their } \\
\text { pharmacist to put their tablets inside a special identifier capsule, or for patients to use } \\
\text { special versions of their pills that are manufactured to incorporate an ingestible chip) }\end{array}$ \\
\hline HealthStats BPro 61 & A watch-like device & Ambulatory blood pressure using applanation tonometry \\
\hline
\end{tabular}

ECG = echocardiography. 
that vital signs are independent variables, the subtle interactions between them can be assessed in a probabilistic manner to determine earlier whether a patient is clinically deteriorating. An example of such a system has been trialled on a surgical stepdown unit in the USA ${ }^{27}$ and was found to be effective in predicting physiological instability before it became overt.

Trends of the vital signs over time ${ }^{28,29}$ or statistical derivatives $^{30}$ can also be used to improve alerting algorithms. Alerting thresholds can be personalised on a patient-by-patient basis using short-term trends ${ }^{31}$ or historical steady-state values. With continuous data, as might be provided by wearable monitors, it is possible to use the recorded waveforms to derive parameters with prognostic significance, such as heart-rate variability, ${ }^{32}$ ECG dispersion ${ }^{33}$ or the entropy of the signal. ${ }^{34}$

In the future, it is likely that computerised algorithms will be used to augment or replace current early warning scores. It might be that, rather than one algorithm reporting a patient's status, multiple complementary algorithms will be used simultaneously, each reporting on the patient's condition from a different perspective. This should resolve the tension between providing a probability of imminent death (NEWS and its precursor ViEWS $)^{35}$ and providing early warning of abnormal physiology to enable timely intervention. ${ }^{36,37}$

\section{Alerting}

Electronic acquisition and storage of observations provides further potential for benefit through the generation of 'smart alerts'. The most commonly used alerting technology on the general ward today is the local single parameter alert, whereby any one of the vital signs breaching a pre-set threshold causes the bedside monitor to sound an audible alarm. Unfortunately, this approach usually leads to a large number of false alarms, partly because the thresholds are often left at default settings, irrespective of the condition of the patient who is being monitored, and partly because signal artefact is often misinterpreted by the monitor. One study in an operating theatre environment showed that $75 \%$ of alarms were spurious and only $3 \%$ indicated serious patient risk..$^{38}$ In an environment where patients move freely, the false alarm rate can be even higher. The problem is exacerbated because the audible alarms from patient monitors are just one group of a host of audible alarms emitted from ward devices, ranging from inflatable mattresses to patient call bells. This quickly leads to so-called 'alarm fatigue', which causes staff to cease responding to alarms in a timely fashion. In the most extreme cases, this can lead to missing the fact that a monitored patient is dying. ${ }^{39}$

Intelligent alerting systems might help to address this problem. These might either generate alarms based on multiple parameters, in a similar fashion to an early warning score, or suppress alarms until a single parameter has consistently exceeded a pre-set threshold, thereby avoiding the problem of alarming owing to transient episodes of artefact.

Computer-generated alarms need not sound at the bedside; they can be delivered remotely to a nursing station, as happens in many level 2 and level 3 areas, or they can be linked directly to the clinicians' pagers. One study on an orthopaedic ward found that sending alerts from pulse oximeters directly to medical emergency teams led to a significant reduction in number of intensive care unit admissions, despite the fact that the thresholds for alerting were set to an arterial oxygen saturation of less than $80 \%$ and a heart rate of under 50 beats per minute or over 140 beats per minute. ${ }^{40}$ Alerting can be based on the hospital early warning score and the same abnormal score can be used to generate multiple alerts that are sent in a sequential fashion to different clinical team members depending on the severity of the abnormality and the response times. A study of one such system suggested that this leads to clinical benefit, although the results were not definitive. ${ }^{41}$ Such systems might help avoid the problem of ward staff recognising that a patient is deteriorating but not calling for help owing to sociocultural factors. ${ }^{42}$

Other approaches to remote alerting involve setting up a centralised monitoring room where patients attached to telemetry devices are surveyed by monitor technicians who call the ward staff via a dedicated hotline if they identify important changes in a patient's condition. ${ }^{43}$ Remote surveillance of patients could also be used to identify patients before they are escalated ${ }^{44}$ or as part of a handover process, such as occurs to a hospital-at-night team.

\section{Potential for benefit}

The concepts outlined in this article have been discussed in the literature for over two decades. During that time, significant progress has been made in the implementation of these ideas. Further refinement is especially needed in algorithms for detecting patients who are deteriorating, along with better methods for assessing their merits. Endpoints that have been previously used, such as length of stay, intensive care unit transfer and mortality, are influenced by a range of factors that can lead to erroneous characterisation of the effectiveness of the systems under study ${ }^{37}$ and make it difficult to make rational choices about the best algorithms to use. ${ }^{7}$

Realisation of benefit is strongly dependent on the quality of the implementation, ${ }^{45}$ user acceptance and the cultural and organisational factors surrounding the introduction of these systems. ${ }^{46}$ Poor implementation not only fails to result in benefit, but can also make care worse. ${ }^{47-50}$

Considerations surrounding the implementation of a technological solution should not be restricted to the technology and the infrastructure that directly supports it. Each technology is supported by a plethora of other systems and the impact on each of these must be assessed. For instance, according to the report that describes it, NEWS will result in an increased alert rate in many hospitals if the suggested triggers for escalation are used. One estimate, based on a database of vital signs from a mixed medical and surgical population, is that in a 1,000-bed hospital, 500 'amber' alerts and 200 'red' alerts would be generated per day. ${ }^{51}$ This might necessitate reorganisation of existing response teams or deployment of extra resources to ensure that the alerts are addressed in an effective and timely manner. Organisations 
that introduce NEWS without providing capable response mechanisms risk the alerts being ignored on occasion, with all the problems that this entails.

Care must also be exercised in identifying the assumptions made when justifying the introduction of new technology. There is yet to be any conclusive evidence that early warning scores improve patient outcomes, ${ }^{52}$ or that minimising errors in recording observations or calculating early warning scores has any significant clinical impact. That these process changes will be beneficial might seem to be self-evident until one considers that nurses frequently rely on cues other than the observations chart to determine whether a patient is deteriorating $^{53}$ and use the observations to support their clinical intuition. Introduction of poorly designed systems can discourage nurses from utilising their clinical impressions because there is nowhere to record them or clinical judgement is removed from the escalation process. ${ }^{25}$

\section{Conclusion}

There are a vast array of technologies, currently available and in development, that promise to improve patient care significantly. Those with the greatest potential to bring about improvement are the systems that electronically store patients' observations and make them available for further processing. Such systems offer benefits both directly, by addressing flaws in the recording process, and also by acting as a foundation upon which other safety systems, such as computerised algorithms and remote alerting systems, can be deployed. For full realisation of the clinical benefits, great care must be taken in the implementation of these technologies, because poor-quality deployment could lead to worse patient care.

\section{References}

1 Vincent C, Neale G, Woloshynowych M. Adverse events in British hospitals: preliminary retrospective record review. BMJ 2001;322:517-9.

2 Hillman KM, Bristow PJ, Chey T et al. Antecedents to hospital deaths. Intern Med J 2001;31:343-8.

3 Hodgetts TJ, Kenward G, Vlackonikolis I et al. Incidence, location and reasons for avoidable in-hospital cardiac arrest in a district general hospital. Resuscitation 2002;54:115-23.

4 Cullinane M, Findlay GP, Hargraves C, Lucas S. An acute problem? London: National Confidential Enquiry into Patient Outcome and Death, 2005.

5 National Institute of Clinical Excellence. Acutely ill patients in hospital. London: NICE, 2007.

6 Findlay GP, Shotton H, Kelly K, Mason M. Time to intervene. London: National Confidential Enquiry into Patient Outcome and Death, 2012.

7 Royal College of Physicians. National early warning score (NEWS). London: Royal College of Physicians; 2012.

8 Capsule Neuron, 2013. www.capsuletech.com/medical-data-collection. htm [Accessed 4 April 2013].

9 Watkinson PJ, Barber VS, Price JD et al. A randomised controlled trial of the effect of continuous electronic physiological monitoring on the adverse event rate in high-risk medical and surgical patients. Anaesthesia 2006;61:1031-9.

10 Bonnici T, Orphanidou C, Vallance D et al. Testing of wearable monitors in a real-world hospital environment: what lessons can be learnt? In Ninth international conference on wearable and implantable body sensor networks, 2012. Washington DC: IEEE, 2012; 79-84.
11 Steventon AA, Bardsley MM, Billings JJ et al. Effect of telehealth on use of secondary care and mortality: findings from the Whole System Demonstrator cluster randomised trial. BMJ 2012;344:e3874-4.

12 Coyle S, Lau KT, Moyna N et al. BIOTEX: biosensing textiles for personalised healthcare management. IEEE Trans Inform Technol Biomed 2010;14:364-70.

13 Kim D-H, Lu N, Ma R et al. Epidermal electronics. Science 2011;333:838-43.

14 Patel S, Park H, Bonato P et al. A review of wearable sensors and systems with application in rehabilitation. J NeuroEngineering Rehabil 2012;9:21.

15 Wu H-Y, Rubinstein M, Shih E et al. Eulerian video magnification for revealing subtle changes in the world. ACM Trans Graphics: SIGGRAPH 2012 Conf Proc 2012;31:65.

16 Poh M-Z, McDuff DJ, Picard RW. Advancements in noncontact, multiparameter physiological measurements using a webcam. IEEE Trans Biomed Eng 2011;58:7-11.

17 Droitcour AD, Seto TB, Park B-K et al. Non-contact respiratory rate measurement validation for hospitalized patients. Conf Proc IEEE Eng Med Biol Soc 2009;2009:4812-5.

18 Sharifahmadian E, Ahmadian A. Adaptive signal processing algorithm for remote detection of heart rate (HR) using ultra-wideband waveforms based on principal component analysis. Conf Proc IEEE Eng Med Biol Soc 2009;2009:5717-20.

19 Prytherch DR, Smith GB, Schmidt PE et al. Calculating early warning scores - a classroom comparison of pen and paper and hand-held computer methods. Resuscitation 2006;70:173-8.

20 Edwards M, McKay H, Van Leuvan C, Mitchell IA. Modified early warning scores: inaccurate summation or inaccurate assignment of score? Critical Care 2010;14:S88.

21 Smith AFA, Oakey RJR. Incidence and significance of errors in a patient 'track and trigger' system during an epidemic of Legionnaires' disease: retrospective casenote analysis. Anaesthesia 2006;61:222-8.

22 Black AD, Car J, Pagliari C et al. The Impact of eHealth on the quality and safety of health care: a systematic overview. PLoS Med 2011;8:e1000387.

23 Van Der Meijden MJ, Tange HJ, Troost JJ, Hasman AA. Determinants of success of inpatient clinical information systems: a literature review. J Am Med Inform Assoc 2003;10:235-43.

24 Berg M. Implementing information systems in health care organizations: myths and challenges. Int J Med Inform 2001;64:143-56.

25 Mackintosh N, Rainey H, Sandall J. Understanding how rapid response systems may improve safety for the acutely ill patient: learning from the frontline. BMJ Qual Saf 2012;21:135-44.

26 Escobar GJ, LaGuardia JC, Turk BJ et al. Early detection of impending physiologic deterioration among patients who are not in intensive care: development of predictive models using data from an automated electronic medical record. J Hosp Med 2012;7:388-95.

27 Hravnak MT, Devita MA, Clontz A et al. Cardiorespiratory instability before and after implementing an integrated monitoring system. Crit Care Med 2011;39:65-72.

28 Ansermino JM, Daniels JP, Hewgill RT et al. An evaluation of a novel software tool for detecting changes in physiological monitoring. Anesth Analg 2009; 108:873-80.

29 Apiletti D, Baralis E, Bruno G, Cerquitelli T. Real-time analysis of physiological data to support medical applications. IEEE Trans Inform Technol Biomed. 2009 May;13:313-21.

30 Hug CW. Detecting hazardous intensive care patient episodes using realtime mortality models. Cambridge: Massachusetts Institute of Technology, 2009.

31 Zhang Y, Szolovits P. Patient-specific learning in real time for adaptive monitoring in critical care. J Biomed Inform 2008;41:452-60. 
32 Ahmad S, Tejuja A, Newman KD et al. Clinical review: a review and analysis of heart rate variability and the diagnosis and prognosis of infection. Crit Care 2009;13:232.

33 Kellett J, Emmanuel A, Rasool S. ECG dispersion mapping predicts clinical deterioration, measured by increase in the Simple Clinical Score. Acute Med 2012;11:8-12.

34 Seely AJE, Macklem PT. Complex systems and the technology of variability analysis. Crit Care 2004;8:R367-84.

35 Prytherch DR, Smith GB, Schmidt PE, Featherstone PI. ViEWS towards a national early warning score for detecting adult inpatient deterioration. Resuscitation 2010;81:932-7.

36 Morgan RJM, Wright MM. In defence of early warning scores. Br J Anaesth 2007;99:747-8.

37 Tarassenko L, Clifton DA, Pinsky MR et al. Centile-based early warning scores derived from statistical distributions of vital signs. Resuscitation 2011;82:1013-8.

38 Block FEF, Schaaf CC. Auditory alarms during anesthesia monitoring with an integrated monitoring system. Ann Biomed Eng 1996;13:81-4.

39 Wallis L. Alarm fatigue linked to patient's death. Crit Care Med 2010;110:16.

40 Taenzer AH, Pyke JB, McGrath SP, Blike GT. Impact of pulse oximetry surveillance on rescue events and intensive care unit transfers: A before-and-after concurrence study. Anesthesiology 2010;112:282.

41 Jones S, Mullally M, Ingleby S et al. Bedside electronic capture of clinical observations and automated clinical alerts to improve compliance with an Early Warning Score protocol. Crit Care Resusc 2011;13:83-8.

42 Shearer B, Marshall S, Buist MD et al. What stops hospital clinical staff from following protocols? An analysis of the incidence and factors behind the failure of bedside clinical staff to activate the rapid response system in a multi-campus Australian metropolitan healthcare service. BMJ Qual Saf 2012;21:569-75.

43 Cale D-D. A new perspective on patient monitoring. Nurs Manage 2007;38:24-6.

44 Smith GB, Prytherch DR, Schmidt PE et al. Hospital-wide physiological surveillance-a new approach to the early identification and management of the sick patient. Resuscitation 2006;71:19-28.

45 Wager KAK, Schaffner MJM, Foulois BB et al. Comparison of the quality and timeliness of vital signs data using three different dataentry devices. Comput Inform Nurs 2010 Jan 1;28:205-12.

46 Delone WH, McLean ER. The DeLone and McLean model of information systems success: a ten-year update. J Manage Inform Syst 2003;19:9-30.
47 Kossman SP, Scheidenhelm SL. Nurses' perceptions of the impact of electronic health records on work and patient outcomes. Comput Inform Nurs 2008;26:69-77.

48 Han YY, Carcillo JA, Venkataraman ST et al. Unexpected increased mortality after implementation of a commercially sold computerized physician order entry system. Pediatrics 2005;116:1506-12.

49 Stevenson JE, Nilsson GC, Petersson GI, Johansson PE. Nurses' experience of using electronic patient records in everyday practice in acute/inpatient ward settings: a literature review. Health Inform J 2010;16:63-72.

50 Yeung MS, Lapinsky SE, Granton JT et al. Examining nursing vital signs documentation workflow: barriers and opportunities in general internal medicine units. J Clin Nurs 2012;21:975-82.

51 Greengross P, Beaumont K, Killen R. Re: a national early warning score for acutely ill patients. BMJ 2012;345:e5310.

52 Gao H, McDonnell A, Harrison DA et al. Systematic review and evaluation of physiological track and trigger warning systems for identifying at-risk patients on the ward. Intensive Care Med 2007;33:667-79.

53 Donohue LA, Endacott R. Track, trigger and teamwork: communication of deterioration in acute medical and surgical wards. Intensive Crit Care Nurs 2010;26:10-7.

54 Intelesens Aingeal, 2013. www.intelesens.com/hospitalmonitoring/ aingeal.html [Accessed 4 April 2013].

55 Equivital, 2013. www.equivital.co.uk/products/tnr/sense-and-transmit [Accessed 4 April 2013].

56 Sotera VisiMobile, 2013. www.visimobile.com/ [Accessed 4 April 2013].

57 Zephyr BioHarness, 2013. www.zephyr-technology.com/products/bioharness_bt [Accessed 4 April 2013].

58 Nonin WristOx2 3150, 2013. www.nonin.com/PulseOximetry/WristWorn/WristOx2-Model-3150 [Accessed 4 April 2013].

59 Isansys LifeTouch Monitor, 2013. www.isansys.com/en/lifetouch_ monitor [Accessed 4 April 2013].

60 Proteus Digital Health, 2013. http://proteusdigitalhealth.com/ [Accessed 4 April 2013].

61 HealthSTATS BPro, 2013. www.healthstats.com/en/bpro.html [Accessed 4 April 2013].

Address for correspondence: Dr T Bonnici, Radcliffe

Department of Medicine, 5th Floor, John Radcliffe Hospital, Headley Way, Headington, Oxford OX3 9DU.

Email: timothy.bonnici@ndm.ox.ac.uk 